\title{
3D FSS with multiple transmission zeros and pseudo elliptic response
}

\author{
Bimal Raj Dutta ${ }^{1}$, Binod Kumar Kanaujia ${ }^{2}$, Chhaya Dalela ${ }^{3}$ \\ ${ }^{1}$ Department of Electronics \& Communication, ANA College of Engineering and Management Studies, India \\ ${ }^{2}$ School of Computational\& Integrative Sciences, Jawaharlal Nehru University, India \\ ${ }^{3}$ Department of Electronics and Communication, JSS Academy of Technical Education, India
}

\begin{tabular}{l} 
Article Info \\
\hline Article history: \\
Received Mar 9, 2019 \\
Revised May 11, 2019 \\
Accepted May 26, 2019 \\
\hline
\end{tabular}

\section{Keywords:}

Frequency selective surface (FSS)

Microstrip line

Multiple transmission zeros

Pseudo-elliptic response

Perfect electric conductor

(PEC)

Perfect magnetic conductor

(PMC)

Resonators

\begin{abstract}
The three-dimensional frequency selective surface (3D FSS) with band reject multiple transmission zeros and pseudo-elliptic response is designed from two-dimensional (2D) periodic array of shielded micro strip lines to realize wide out-of-band radio wave rejection. The 3D FSS array consists of multimode cavities whose coupling with air can be controlled to obtain a desired frequency range. The proposed FSS with shorting via to ground exhibits pseudo-elliptic band-reject response in the frequency range from $6 \mathrm{GHz}$ to $14 \mathrm{GHz}$. As the plane wave of linear polarization incidents perpendicularly to the shielded micro strip line with perfect electric conductor (PEC) and perfect magnetic conductor (PMC) boundary walls, two quasi-TEM modes are obtained known as air mode and substrate mode. The first 3D FSS design is a combination of two or more resonators. Furthermore, second 3D FSS design with three shorting vias result more elliptic band reject frequency response and a pass band transmission pole. All in phase resonators of design give transmission poles and out of phase combination of resonators give transmission zeros respectively. The proposed 3D FSS is designed and simulated using Ansys HFSS software. These designs exhibit an improved performance for many practical applications such as antenna sub-reflector, and spatial filters.
\end{abstract}

Copyright $@ 2019$ Institute of Advanced Engineering and Science. All rights reserved.

\section{Corresponding Author:}

Bimal Raj Dutta,

Department of Electronics and Communication,

ANA College of Engineering and Management Studies, India.

Email: brajdutta@gmail.com

\section{INTRODUCTION}

Unlike the conventional filters, frequency selective surfaces (FSS) are the spatial filters for the electromagnetic waves. This has been the topic of research for the past four to five decades and used in many applications such as antenna sub-reflectors, spatial filters with band pass or band stop characteristics, polarizer etc. [1,2]. Traditional two-dimensional 2D FSS are designed by a 2D periodic array of unit cells that are either slotted elements etched out of a conductive surface or printed elements on a dielectric surface [3, 4]. Single layer of such traditional 2D FSS suffers from poor filtering response, poor selectivity, unstable angular response and narrow bandwidth. On cascading number of these layers with a dielectric material sandwich in between each of them, a significant improved filtering response has been obtained [5]. That resulted in a small increase in bandwidth in past. These designs followed Butterworth or Chebyshev filtering response with lack of elliptic response. Also these conventional multilayer design filters did not obtain wide out-of-band frequency rejection. The 3D FSS, Elliptic filtering response has been achieved by the increase of level of cross-coupling between the resonators which was not obtained in cascaded 2D FSS designs, even though they had used direct-coupled resonators [6]. Elliptic filtering response can also be achieved by making source/load coupled with more than one resonator [7] as shown in Figure 1. 


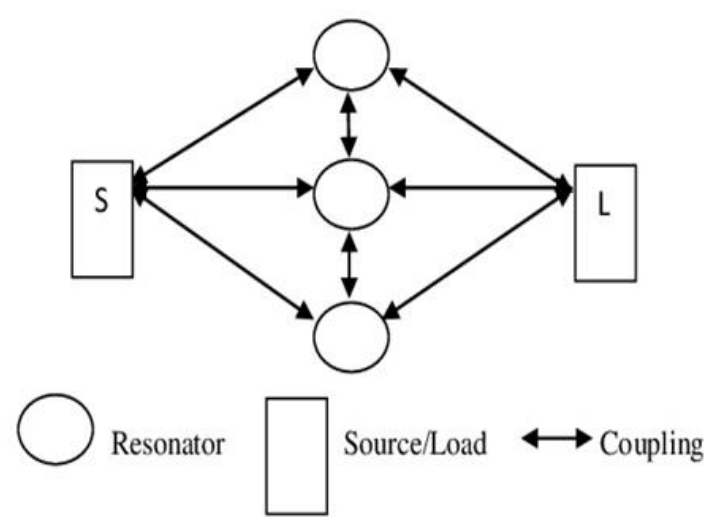

Figure 1. Multiple resonators and cross-coupling between source and load to obtain transmission poles/zeros at finite frequencies

With this new concept of 3D frequency selective surface, multiple transmission poles/zeros will be produced through multimode resonators. A case of band-reject pseudo-elliptic filtering response with dualband rejection and broadband rejection has been thoroughly studied for planar micro strip filters [8]. By introducing transmission zeros at desired finite frequencies, wide band rejection filters will be realized. This 3D FSS consists of a 2D periodic array of shielded micro strip lines placed vertically with support of two quasi-TEM modes namely the air mode and substrate mode. These modes are generated when a linearly polarized plane wave is incident perpendicularly to the micro strip at the air-to-micro strip line discontinuity [9].

In this paper two 3D FSS designs have been presented with the first design of dual-band band-reject characteristics having a shielded micro strip lines with PEC and PMC boundary wall along with a single shorting via that constitutes of total three resonators (one air mode resonator and two substrate mode resonators) as shown in Figure $2[10,11]$. The second design is of wide band-reject characteristics which are geometrically modified from the first design with the inclusion of three more shorting via those results in the increase of the band-reject frequency [12,13]. This increase is due to the addition of more number of shorting via at the centre of the micro strip.
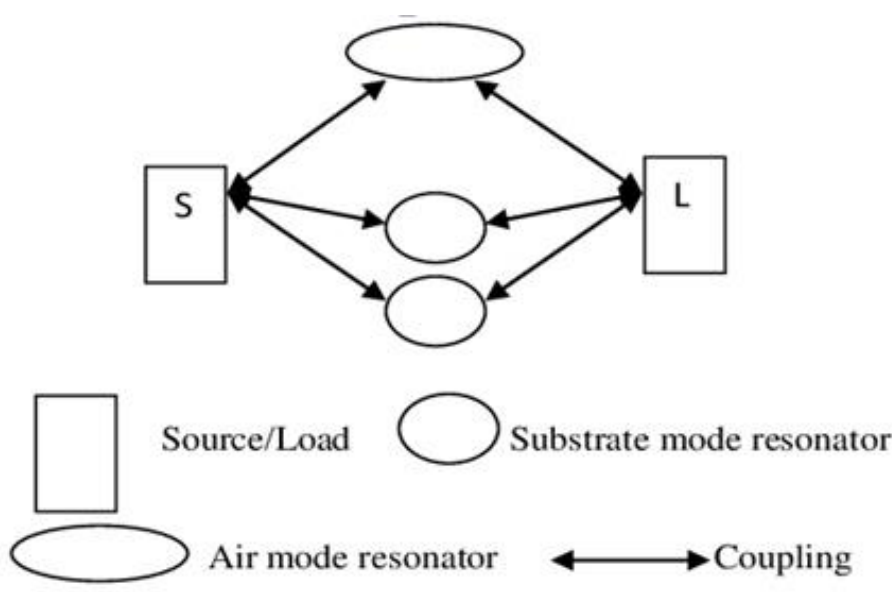

Figure 2. 3D FSS with single air mode resonator and two substrate mode resonator

\section{RESULTS AND DISCUSSION}

The 3D FSS equivalent model represents in Figure 3. For simplification we consider a model of four resonators $R_{1}, R_{2}, R_{3}$ and $R_{4}$, those are linked with input and output port, whereas resonators $R_{a}$ and $R_{b}$ are linked only to input port. The resonators $R_{c}$ and $R_{d}$ are linked to output port only. Each resonator represents a propagating mode that provide either a transmission zero or transmission pole at their respective resonant 
frequencies. Resonator $R_{a}, R_{b}, R_{c}$ and $R_{d}$ provide additional transmission zeros at the desired frequencies thus increasing the bandwidth frequency of a band-stop FSS or improving the selectivity of a band-pass FSS. This 3D FSS design provides flexibility in producing desired number of transmission zeros/poles at finite frequencies by controlling the number of resonators and their resonance frequencies, therefore 3D FSS have quasi-elliptic filtering response [9].2D FSS are the special case of the equivalent model of 3D FSS in Figure 3. It consists of only one resonator or mode that provides a single transmission zero/pole, which leads to poor filtering performance. Principle of operation of this 3D FSS design needs to be reviewed to understand how the resonators are formed. When a plane wave of TE polarization oriented perpendicular to the shielded micro strip incidents at the air-to-micro strip line discontinuity, two propagating modes are created namely air and substrate modes [8]. These modes link input and output ports. At low frequencies the incident signals travel through the air path from one port to other providing low-pass response, but as the frequency of operation increases the same signal travels through two paths (air and substrate). The substrate mode resonator will always resonate first than the air mode resonator. As the guided wavelength $\left(\lambda_{g}\right)$ of the substrate mode is less than the wavelength $\left(\lambda_{0}\right)$ of air mode. In such propagating modes of air and substrate, there are two possibilities; (a) the first being the generation of transmission poles which is produced whenever the electrical length of any resonator becomes equal to $\pi$, (b) the second being the generation of transmission zeros which is produced whenever the phase difference between signals in the air and substrate path is $180^{\circ}$.

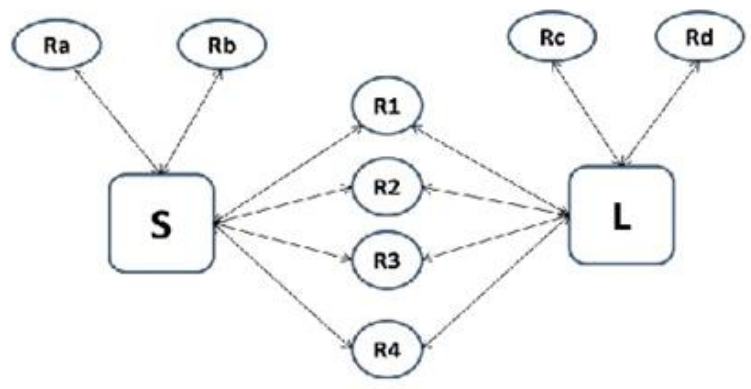

Figure 3. Equivalent model of a 3D FSS having multiple resonators

There are two modes present in the equivalent model as shown in Figure 4. The two transmission poles are obtained due to the two resonators $\left(R_{1}, R_{2}\right)$, which are resonating at their resonant frequencies. Also one transmission zero is obtained at a frequency where the two modes are $180^{\circ}$ out of phase.

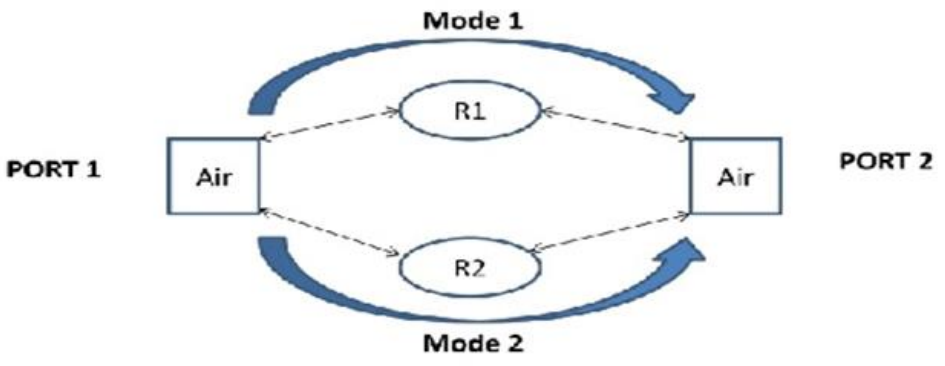

Figure 4. Equivalent model of a 3D FSS having two resonators/modes

\section{FIRST DESIGN (BANDSTOP FSS)}

\subsection{Structure description}

A three dimensional view of a 3D band-reject FSS is shown below in the Figure 5(a) and Figure 5(b). It is a 2D periodic array combination of shielded micro strip placed vertically in $\mathrm{z}$ direction. 
The array shown in Figure 5(a) is a combination of $4 \times 4$ unit cells. Each unit cell as shown in fig $6 \mathrm{a}$ has the height in y direction $h=3.524 \mathrm{~mm}$, which is a combination of air and substrate region. The height of the substrate in $\mathrm{y}$ direction is $d=1.524 \mathrm{~mm}$. The length of the unit cell/micro strip line in $\mathrm{z}$ direction is $l=10.5 \mathrm{~mm}$. The width of the unit cell/substrate in $\mathrm{x}$ direction is $b=5 \mathrm{~mm}$. The substrate material incorporated in the design is Rogers RO3003 having dielectric constant of v.

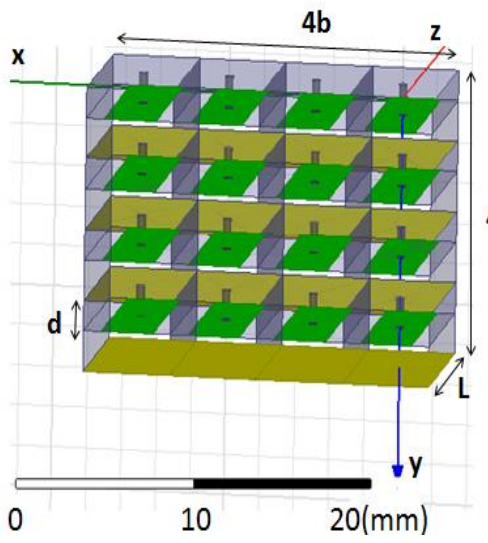

(a)

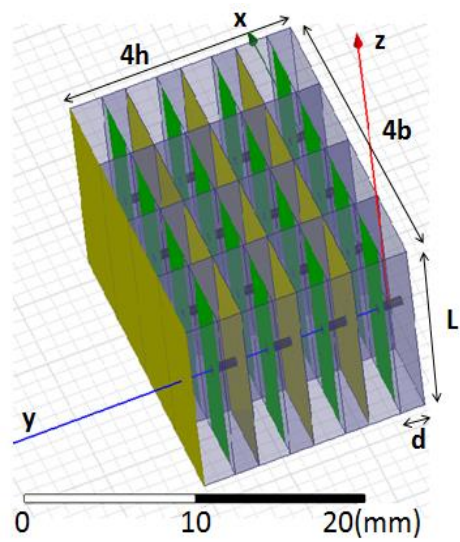

(b)

Figure 5.Three-dimensional views of the band stop FSS (a) side view(b) top view

The width of the micro strip line in $\mathrm{x}$ direction is $w=3 \mathrm{~mm}$ shown in the Figure 6(a). The design also includes a shorting via of diameter $D=0.5 \mathrm{~mm}$ that connects ground to the centre of micro strip line. The Unit cell reappears after a period of " $b$ " in $\mathrm{x}$ direction and " $\mathrm{h}$ " in y direction. The designed 3D FSS have a PEC boundary in $\mathrm{X}-\mathrm{z}$ plane and PMC boundary $\mathrm{y}-\mathrm{z}$ plane. A plane wave with TE polarization travelling in $\mathrm{z}$ direction will be incident at port 1 (air to micro strip line discontinuity) as shown in Figure 6(b).

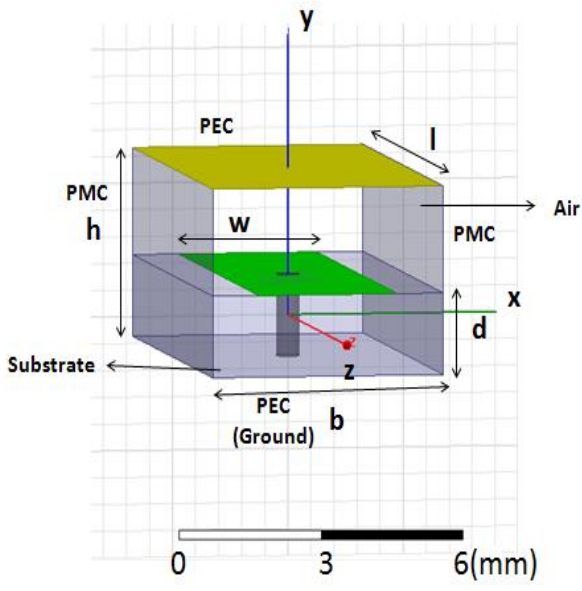

(a)

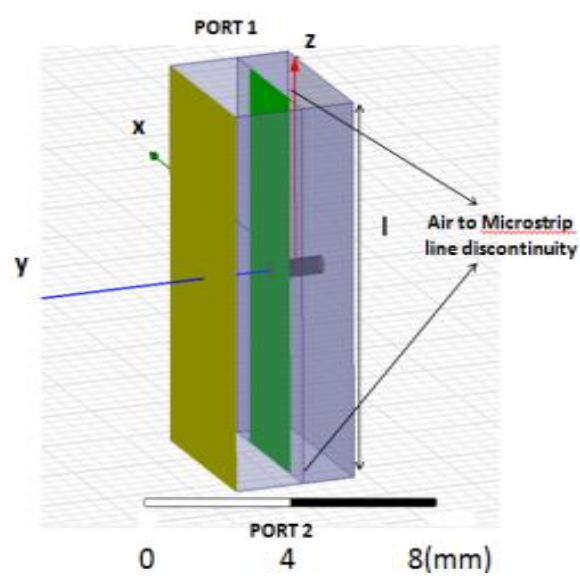

(b)

Figure 6. Three-dimensional view of a unit cell of (a) top view (b) side view

\subsection{Analysis of simulated result for 3D bandstop FSS}

The simulated S parameter results are shown in Figure 7. This can be used to analyse the band stop characteristics of the designed 3D FSS. 


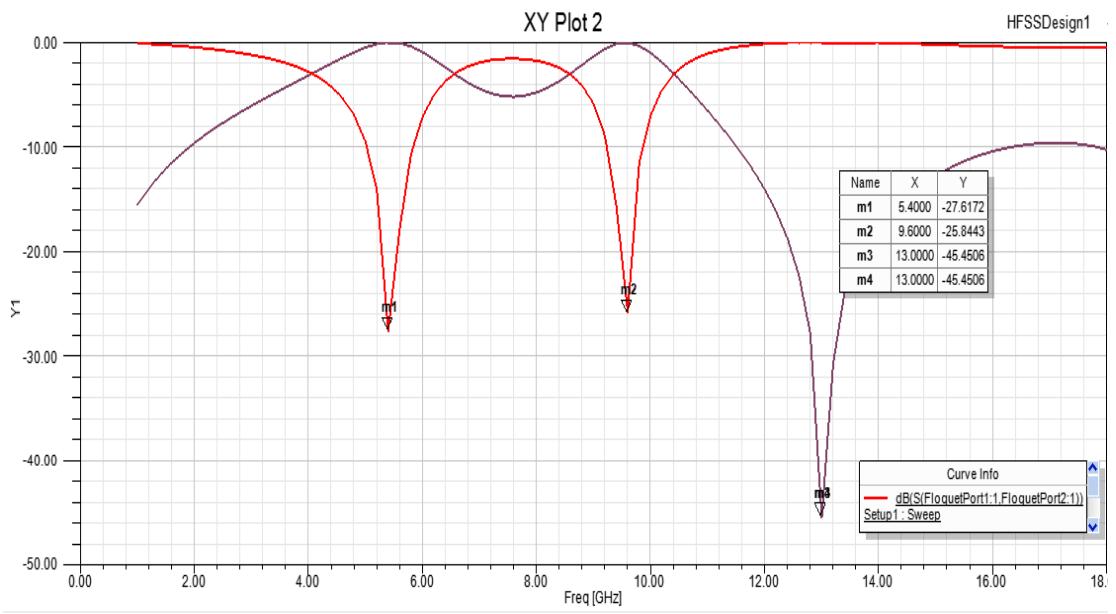

Figure 7. Simulated S parameter results from HFSS for the bandstop FSS

A combination of $S_{11}$ (Reflection Coefficient) and $S_{21}$ (Transmission Coefficient) parameters are shown in Figure 7. These are the simulated results of the equivalent two port device of a unit cell. Here Floquet port excitation has been provided in $\mathrm{z}$ direction of port 1 and port 2 . It results in three resonance frequencies. The two of them are the transmission zero at frequencies $5.4 \mathrm{GHz}$ and $9.6 \mathrm{GHz}$ due to substrate mode resonators. The third one is transmission pole at frequency $13 \mathrm{GHz}$ due to air mode resonator. It is observed that the band-reject frequency of the design 3D FSS has been increased by the addition of an extra transmission zero, which is obtained by the inclusion of shorting via. This short via introduced an extra shortcircuit resonator in addition to existing open-circuit micro strip line resonator [10].

\subsection{Equivalent circuit model analysis}

The unit cell of the 3D FSS is resulted due to the combination of two sub networks i.e. air path network and substrate path network. Each section of these sub networks is represented by the transfer matrix. When each section transfer matrix of air path sub network is cascaded, the final transfer matrix of air path sub network will result as shown in the below given Figure 8(a).

The A, B, C, D parameters of the transfer matrix are used to realize the impedance parameters of the impedance matrix $[Z]$. Thereby with the aid of impedance parameters, the scattering parameters of the $[S]$ matrix are realized $[10,11]$. The A, B, C, D parameters of the transfer matrix of the Figure 7 sub network depend on the equivalent lumped Capacitor $\left(C_{a}\right)$, transmission line parameter i.e. characteristic impedance of the air path $\left(Z_{a}\right)$ and the electrical length of the air path $\left(\Theta_{a}\right)$. The capacitor $C_{a}$ is used to represent the discontinuity between the air region of the unit cell and the air region outside the unit cell. The block diagram of the substrate path sub network as per Figure 8(b), represents the transfer matrix of each section, an overall transfer matrix of the substrate path network is resulted due to cascading of the each block.

The A, B, C, D parameters of the transfer matrix of the substrate path sub network shown in Figure $8 \mathrm{~b}$, depend on the following (a) two equivalent lumped elements i.e. Capacitor $\left(C_{s}\right)$ and Inductor $\left(L_{s}\right)$ (b) transmission line parameter i.e. characteristic impedance of the substrate path $\left(Z_{s}\right)(\mathrm{c})$ the electrical length of the substrate path $\left(\Theta_{S}\right)$. The capacitor $C_{s}$ is used to represent the discontinuity between the substrate region of the unit cell and the air region outside the unit cell. The inductor $\left(L_{s}\right)$ represents the shorting via that connects ground to the micro strip.

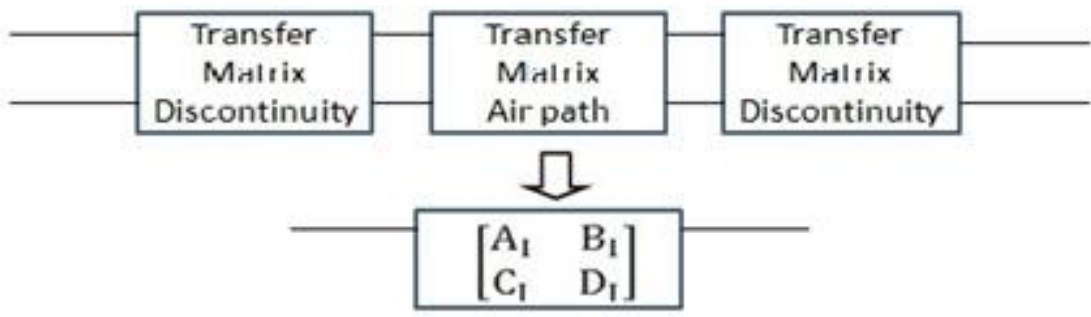

(a) 


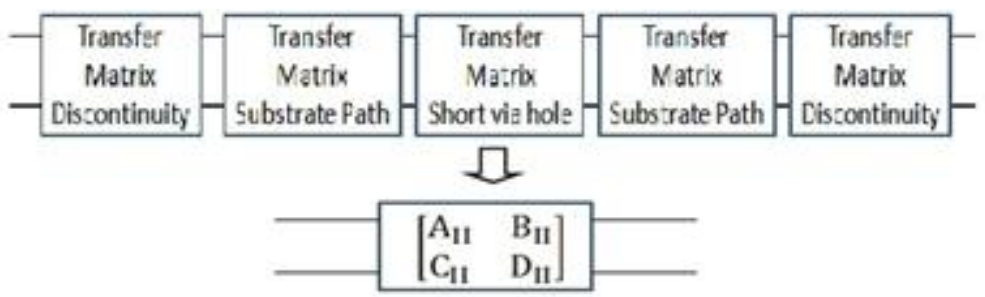

(b)

Figure 8. (a) Block diagram showing the transfer matrix of the first sub network (air path),

(b) Block diagram showing the transfer matrix of the second sub network (substrate path)

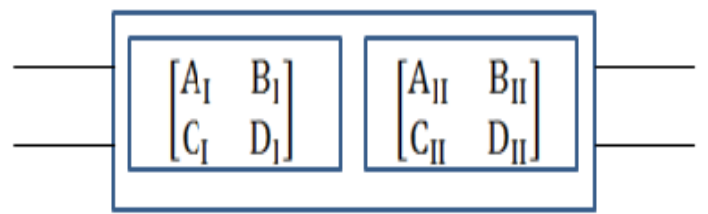

Figure 9. Block diagram showing the cascaded combination of the transfer matrix of the two sub network (air and substrate path)

The equivalent transfer matrix of the two sub network is cascaded as shown above in Figure 9, to realize the impedance parameters of the equivalent circuit model [12] which are as follows;

$$
\begin{aligned}
& Z_{11}=Z_{22}=\frac{A_{I}}{C_{I}}+\frac{A_{I I}}{C_{I I}} \\
& Z_{12}=Z_{21}=\frac{1}{C_{I}}+\frac{1}{C_{I I}}
\end{aligned}
$$

Now the scattering parameters [14] is obtained from the above impedance parameters as follows;

$$
\begin{gathered}
S_{11}=S_{22}=\frac{Z_{11}^{2}-Z_{12}^{2}-Z_{0}^{2}}{\left(Z_{11}+Z_{0}\right)^{2}-Z_{12}^{2}} \\
S_{21}=S_{12}=\frac{2 Z_{12}^{2} Z_{0}}{\left(Z_{11}+Z_{0}\right)^{2}-Z_{12}^{2}}
\end{gathered}
$$

The equivalent lumped elements $C_{a}, C_{s}$ and $L_{s}$ are approximately calculated by the physical dimensions of the unit cell structure as follows;

$$
\begin{aligned}
& C_{a} \approx \frac{w}{b} \frac{h}{\omega(h-d) z_{0}} \tan \Delta \Theta \\
& C_{s} \approx \varepsilon_{r} \frac{w}{b} \frac{h}{\omega d z_{0}} \tan \Delta \Theta \\
& L_{s}=\frac{\mu_{0}}{2 \pi}\left[d \ln 2 \frac{d+\sqrt{\left(\frac{D}{2}\right)^{2}+d^{2}}}{D}+1.5\left(\frac{D}{2}-\sqrt{\left(\frac{D}{2}\right)^{2}+d^{2}}\right)\right]
\end{aligned}
$$

Here $Z_{0}$ is the characteristic impedance of the parallel plate waveguide that represents the free space region outside the unit cell carrying the TE polarized plane wave. The $\tan \Delta \Theta$ is evaluated from [15] as follows;

$$
\tan \Delta \Theta=\frac{2 h}{\lambda}\left(\frac{h-d}{h} \ln \frac{h}{h-d}+\frac{d}{h} \ln \frac{h}{d}\right)+S_{1}\left(\frac{2 h}{\lambda} ; 0,0\right)-S_{1}\left(\frac{2(h-d)}{\lambda} ; 0,0\right)-S_{1}\left(\frac{2 d}{\lambda} ; 0,0\right)
$$


where

$$
S_{1}(x ; 0,0)=\sum_{n=1}^{\infty}\left(\sin ^{-1}\left(\frac{x}{n}\right)-\left(\frac{x}{n}\right)\right)
$$

The propagation constant of the air mode $\beta_{a}$ and the substrate mode $\beta_{s}$ are evaluated from [13], thereby the electrical length of these two modes are realized as follows;

$$
\begin{aligned}
& \text { Air mode } \Theta_{a}=\beta_{a} l \\
& \text { Substrate mode } \Theta_{s}=\beta_{s} l
\end{aligned}
$$

\subsection{Design methodology}

The basic design guidelines for the efficient designed 3D FSS are as follows;

a. The width $b$ and the height $h$ of the unit cell should be much smaller than the operating wavelength (32.6mm). This results in stable frequency response under variation of the angle of incidence $\left(0^{0}-60^{0}\right)$.

b. The value of $\varepsilon_{r}$ plays a vital role in deciding whether the designed 3D FSS is narrowband or wideband FSS. A low value of $\varepsilon_{r}$ results in single transmission zero (narrowband FSS), while large value of $\varepsilon_{r}$ results in two or more transmission zeros (wideband FSS).

c. The length of the micro strip is calculated as;

$$
L=\frac{\lambda g}{2}=\frac{\lambda_{0}}{2 \sqrt{\varepsilon_{r e}}}
$$

Here $\lambda_{g}$ is the guided wavelength at the required center frequency of the FSS and $\varepsilon_{r e}$ is the effective dielectric constant. For the micro strip of thickness $t \rightarrow 0$ and $w / d \geq 1$ the value of $\varepsilon_{r e}$ is evaluated [11] as follows;

$$
\varepsilon_{r e}=\frac{\varepsilon_{r}+1}{2}+\frac{\varepsilon_{r}-1}{2}\left\{\left(1+12 \frac{d}{w}\right)^{-0.5}\right\}
$$

The value of $\varepsilon_{r e}$ is a function of frequency and its value lies between;

$$
1<\varepsilon_{r e}<\varepsilon_{r}
$$

The frequency of operation increases $\varepsilon_{r e}$ approaches $\varepsilon_{r}$.

The diameter $D$ of the short via affects the first resonant frequency, which gets lower as the diameter decreases due to the dependency of the inductance $L_{s}$ on $D$.

\section{SECOND DESIGN (IMPROVED BAND REJECT FSS)}

\subsection{Structure description}

Previously designed 3D band-reject FSS had a dual band band-reject frequency which could not effectively reject the entire required band of frequencies. Also the frequency response achieved was not close to the quasi-elliptic response behaviour. A new 3D FSS has been designed as shown below in Figure 10 (a, b) that overcomes the shortcoming of the previous design.

Physical dimensions of this design are similar to the previous design. Here the array is also a combination of $4 \times 4$ unit cells consisting of a shielded micro strip. The difference is seen in the construction of the unit cell which has four shorting via connecting ground to the micro strip instead of having a single shorting via. All four of these shorting via are placed at an equal distance from each other along the $\mathrm{x}$ axis under the centre of the micro strip as shown in Figure $11(a, b)$. 


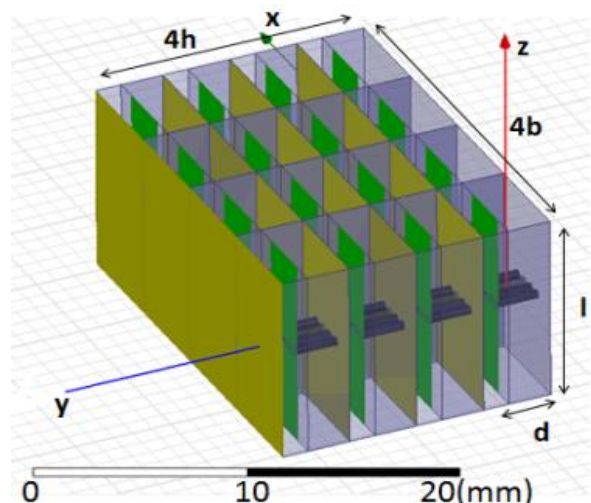

(a)

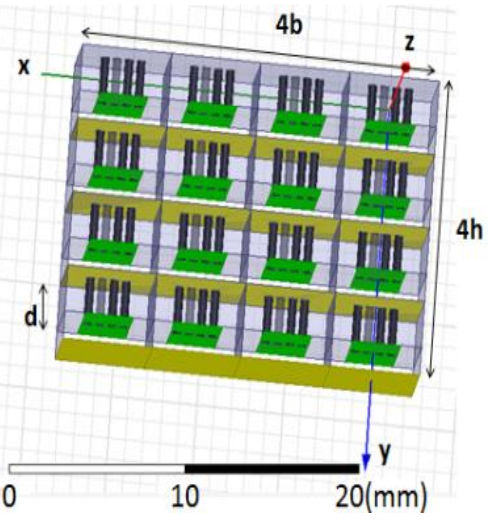

(b)

Figure 10. Three-dimensional view of the improved band stop FSS(a) side view (b) top view

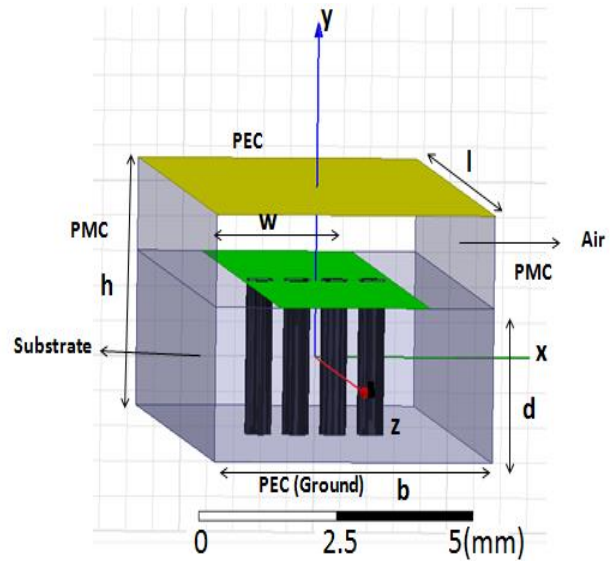

(a)

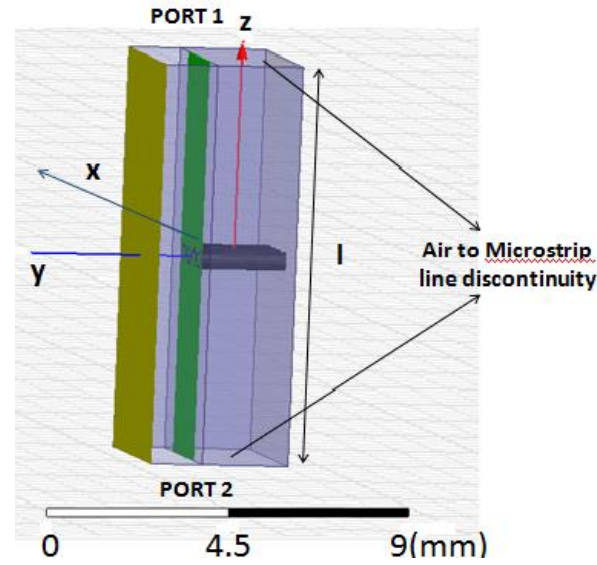

(b)

Figure 11. Three-dimensional view of the unit cell of the improved band stop FSS, (a) top view, (b) side view

\subsection{Analysis of simulated result for 3D bandstop FSS}

The simulated S parameter results as shown in Figure 12, is used to analyse the improved bandreject characteristics of the designed 3D FSS. The graph shown below is a combination of reflection and transmission coefficient $\left(S_{11}, S_{21}\right)$. In this graph also we get three resonant frequencies because the number of resonators is still same as that in the previous design.

Out of these, two transmission zeros are at $7.4 \mathrm{GHz}$ and $9.2 \mathrm{GHz}$ respectively. The third one is the transmission pole at $11.8 \mathrm{GHz}$. The result observed through this graph shows that the whole band of frequencies starting from $6.67 \mathrm{GHz}$ up to $9.62 \mathrm{GHz}$ are rejected out thus improving the band-reject efficiency from the previous design[12] which rejected dual narrowband frequency ranges only. Also it is observed there is a shift in the resonant frequency of the transmission pole towards lower frequency $11.8 \mathrm{GHz}$, which makes the frequency response more elliptical from the previous design. 


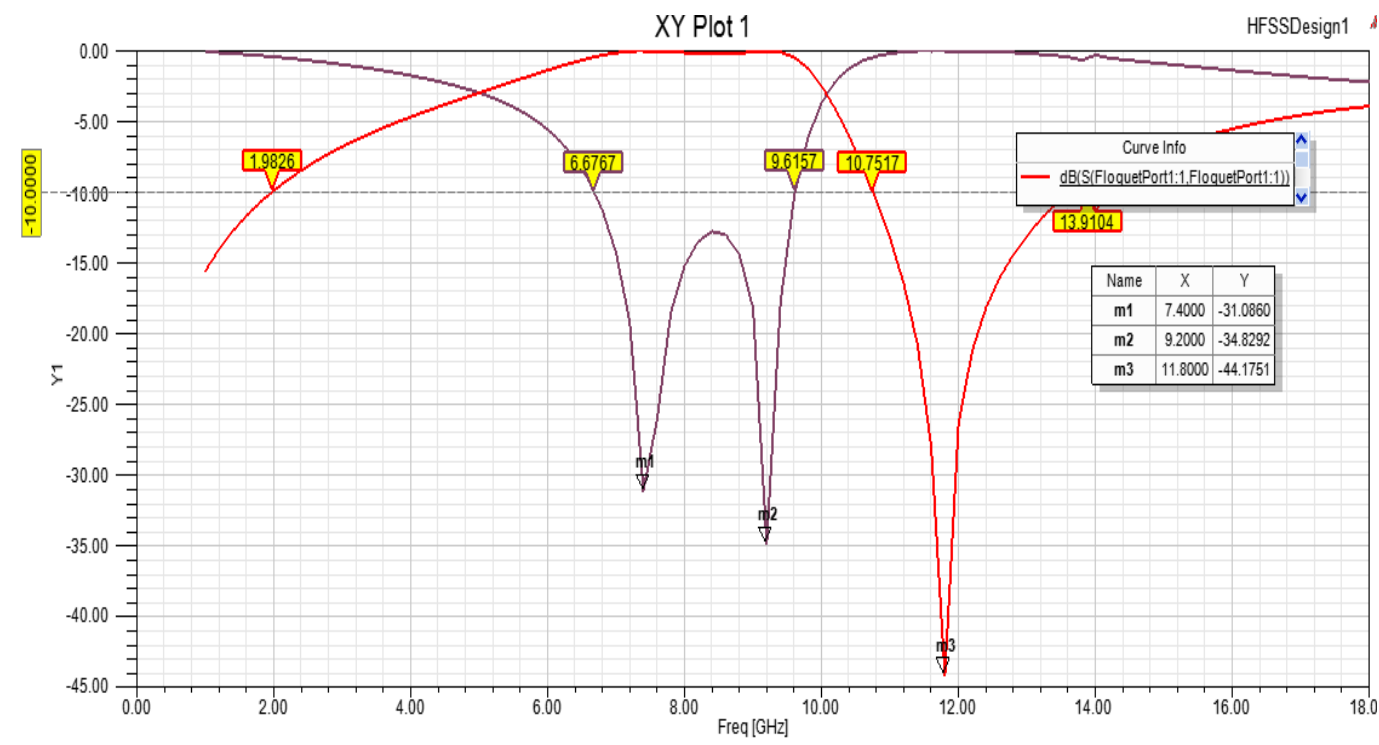

Figure 12. Simulated S parameter results from HFSS for the improved band-reject 3D FSS

Both design1and design2 results are compared in below given Table 1 . The design1 result in Figure 7 shows one transmission zero at $5.4 \mathrm{GHZ}$ with a band rejection from 5.1GHZ to 5.9GHZ (0.8GHZ) and the second transmission zero $9.6 \mathrm{GHZ}$ with a band rejection from $9.2 \mathrm{GHZ}$ to $9.9 \mathrm{GHZ}(0.7 \mathrm{GHZ})$. It has one transmission pole at $13 \mathrm{GHZ}$ with less elliptical response. The design 2 result in Figure 12 shows one transmission zero at $7.4 \mathrm{GHZ}$ and the second transmission zero $9.2 \mathrm{GHZ}$ with a single band rejection from $6.67 \mathrm{GHZ}$ to $9.2 \mathrm{GHZ}$ (3GHZ). It has one transmission pole at $11.8 \mathrm{GHZ}$ with more elliptical response.

Table 1. Comparison between the first and second design

\begin{tabular}{ccc}
\hline Parameters & First Design & Second Design \\
\hline & Dual-band band-reject obtained of & Single \\
Bandwidth of the band-reject FSS & $(1) .0.8 \mathrm{GHz}$ & broad-band band-reject \\
& $(2) .0.7 \mathrm{GHz}$ & obtained of $3 \mathrm{GHz}$ \\
Transmission pole frequency & $13 \mathrm{GHz}$ & $11.8 \mathrm{GHz}$ \\
Elliptical Response & Less elliptical & More elliptical \\
\hline
\end{tabular}

\section{CONCLUSION}

Two 3D band-reject FSS have been presented that are 2D periodic array arrangement of shielded micro strip line. The first design with only single short via shows dual narrowband band-reject 3D FSS with quasi elliptic response. The second design with four short via shows a broadband band-reject 3D FSS with more elliptic response than the former design. Thus 3D FSS allows greater operating bandwidth than 2D FSS with inclusion of more transmission zeros and also improves the selectivity of the operating bandwidth.

\section{ACKNOWLEDGEMENTS}

The writers would like to thank Faculty of Electronics Department of A.N.A College of Engineering and Management Studies for supportive in attaining the information and material in the development for our work. The appreciation also goes to the Dr Binod Kumar kanaujia Dr.Chhaya Dalela, and the Research Scholar from AKTU for guidance and support for this work.

\section{REFERENCES}

[1] B.A.Munk, Frequency Selective Surfaces: Theory and Design. New York, NY, USA: Wiley, 2000

[2] A. K. Rashid, Z. Shen, and B. Li, "An Overview of Three-Dimensional Frequency Selective Structures," IEEE Antennas and Propagation Magazine, Vol. 56, No. 3, June 2014.

[3] Tariq Rahim and Jiaodong $\mathrm{Xu}$ "X-bandBand- pass Frequency Selective Surface for Radome Application"TELKOMNIKA (Telecommunication, Computing, Electronics and Control). Vol. 16, No. 2.November 2015, pp. 281-285 
[4] Umair Rafique, Syed Ahsan Ali, M.Tausif Afzal and Mudassar Abdin “ Bandstop Filter Design for GSM Shielding using Frequency Selective Surfaces" International Journal of Electrical and Computer Engineering Vol.2.NO.6.December 2012pp.846- 850

[5] T.Zhang, H.H.Ouslimani,Y. Letestu, A.LeBayon, andL.R.Darvil, "A low profile multilayer seventh order band-pass frequency selective surface (FSS) for millimeter-wave application," inProc. IEEE $13^{\text {th }}$ Annu. Wireless Microw. Technol. Conf., 2012, pp. 1-4.

[6] Abbas Abbaspour-Tamijani, Kamal Sarabandi "Antenna Arrays as a Class of Bandpass Frequency -Selective Surfaces" IEEE Transaction on Microwave Theory and Techniques, Vol. 52, no. 8, Aug. 2004.

[7] S. Amari, U. Rosenberg, and J. Bornemann, "Adaptive synthesis and design of resonator filters with source/loadmultiresonator coupling,"'IEEE Trans. Microw. Theory Tech., vol. 50, no. 8, pp. 1969-1678, Aug.2002

[8] U. Rosenberg and W. Hagele, "Advanced multimode cavityfilter design using source/load-resonance circuit cross couplings," IEEE Microw. Guided Wave Lett., vol. 2, no. 12, pp. 508-510, Dec. 1992.

[9] W. M. Fathelbab and M. B. Steer, "Parallel-coupled line filters with enhanced stopband performances," IEEE Trans.Microw. Theory Tech. vol.53, no. 12, pp. 3774-3781, Dec. 2005.

[10] A. K. Rashid and Z. Shen, "A novel band-reject frequency selective surface with pseudo-elliptic response,"IEEE Trans. Antennas Propag.,vol. 58, no. 4, pp. 1220-1226, Apr. 2010

[11] A. K. Rashid, Z. Shen, and B. Li, "An elliptical bandpass frequency selective structure based on microstrip lines,"IEEE Trans. Antennas Propag., vol. 60, no. 10, pp. 4661-4669, Oct. 2012

[12] Bo Li and Z. Shen, "Three-dimensional bandpass frequency-selective structures with multiple transmission zeros," IEEE Trans. On Microwave theory and techniques, vol. 61, no. 10, Oct. 2013

[13] A. K. Rashid, Z. Shen, and S. Aditya, "Wideband microwave absorber based on a two-dimensional period array of microstrip lines,"IEEE Trans Antennas Propag., vol. 58, no. 12, pp. 3913-3922, Dec. 2010

[14] J.-S. Hong and M. J. Lancaster, Microstrip Filter for RF/Microwave Applications. New York, NY, USA: Wiley, 2001.

[15] David M. Pozar, “Microwave Engineering”, John Wiley \& Sons,Inc 